\title{
Quantum Simulation Meets Nonequilibrium Dynamical Mean-Field Theory: Exploring the Periodically Driven, Strongly Correlated Fermi-Hubbard Model
}

\author{
Kilian Sandholzer®, ${ }^{1}$ Yuta Murakami, ${ }^{2}$ Frederik Görg, ${ }^{1}$ Joaquín Minguzzi, ${ }^{1}$ Michael Messer, ${ }^{1}$ Rémi Desbuquois, ${ }^{1}$ \\ Martin Eckstein, ${ }^{3}$ Philipp Werner, ${ }^{2}$ and Tilman Esslinger ${ }^{1}$ \\ ${ }^{1}$ Institute for Quantum Electronics, ETH Zurich, 8093 Zurich, Switzerland \\ ${ }^{2}$ Department of Physics, University of Fribourg, 1700 Fribourg, Switzerland \\ ${ }^{3}$ Department of Physics, University of Erlangen-Nürnberg, 91058 Erlangen, Germany
}

(Received 29 November 2018; published 8 November 2019)

\begin{abstract}
We perform an ab initio comparison between nonequilibrium dynamical mean-field theory and optical lattice experiments by studying the time evolution of double occupations in the periodically driven FermiHubbard model. For off-resonant driving, the range of validity of a description in terms of an effective static Hamiltonian is determined and its breakdown due to energy absorption close to resonance is demonstrated. For near-resonant driving, we investigate the response to a change in driving amplitude and discover an asymmetric excitation spectrum with respect to the detuning. In general, we find good agreement between experiment and theory, which cross validates the experimental and numerical approaches in a strongly correlated nonequilibrium system.
\end{abstract}

DOI: 10.1103/PhysRevLett.123.193602

Quantum simulation exploits the high degree of control over a quantum system, such as ultracold atoms, to explore the complexity of many-body physics [1-4]. To gain reliable insights from this approach it is important to benchmark the simulator against numerical or analytical methods. Extensive comparisons have been performed for static systems, such as the Fermi-Hubbard model, which captures essential effects of electronic correlations in solids [5-11]. A new frontier in many-body physics is the study of driven systems, such as high-temperature superconductors exposed to intense laser fields $[12,13]$ or cold atoms in topologically nontrivial band structures $[14,15]$. It is indeed a considerable challenge to understand the consequences of periodic driving, often referred to as Floquet engineering, in correlated lattice models $[15,16]$. An important question is to what extent the properties of the driven system can be captured by an effective static description (Floquet Hamiltonian) despite its nonequilibrium nature. We address this subject by studying the driven Fermi-Hubbard model in the experimental setting of an optical lattice and directly compare the results to nonequilibrium dynamical mean field theory (DMFT).

Effective Floquet Hamiltonians can be derived from high-frequency expansions or time-dependent SchriefferWolff transformations [17-20]. It is expected that these effective models describe the dynamics and thermodynamics of the many-body system under certain conditions. Nevertheless, the real Floquet-engineered state may be characterized by nonthermal energy distributions induced by switch-on procedures or energy absorption from the periodic drive [18,21-25] and higher order corrections. We use ultracold fermions in a brick wall lattice to avoid unwanted excitation processes induced by the driving [26] that otherwise lead to heating of the interacting system [27-32]. A theoretical formalism which captures the full time evolution is nonequilibrium DMFT [33-35]. This method has been used to study a broad range of nonequilibrium setups in single-band [35] and multiband [36] Hubbard models, and to interpret pump-probe experiments on correlated solids at a qualitative level [37]. However, there have been only a few attempts to test the accuracy of this method for the nonequilibrium dynamics in finitedimensional lattices [38] and there has so far been no $a b$ initio comparison to experiments.

We investigate the driven Fermi-Hubbard model

$\hat{H}(t)=-\sum_{\langle\mathbf{i}, \mathbf{j}\rangle, \sigma} J_{\mathbf{i j}} c_{\mathbf{i} \sigma}^{\dagger} c_{\mathbf{j} \sigma}+U \sum_{\mathbf{i}} \hat{n}_{\mathbf{i} \uparrow} \hat{n}_{\mathbf{i} \downarrow}+E(t) \sum_{\mathbf{i}, \sigma} x_{\mathbf{i}} \hat{n}_{\mathbf{i} \sigma}$

on a three-dimensional brick wall lattice structure [Fig. 1(a)]. Here, $\hat{c}_{\mathbf{i} \sigma}^{\dagger}$ and $\hat{n}_{\mathbf{i} \sigma}$ are the fermionic creation and number operators at site $\mathbf{i}=\left(i_{x}, i_{y}, i_{z}\right)$ in spin-state $\sigma=\uparrow, \downarrow$ respectively. The nearest neighbor $\langle\mathbf{i}, \mathbf{j}\rangle$ tunneling rate is denoted by $J_{\mathrm{ij}}$, the onsite interaction by $U$ and the timeperiodic driving field in the $x$ direction by $E(t)$, with $x_{\mathbf{i}}=\langle\hat{x}\rangle_{\mathbf{i}}$ the $x$ position of the Wannier function on site $\mathbf{i}$. Our study covers the off-resonant and near-resonant driving regimes, which are described by different effective Hamiltonians; see Fig. 1(b).

Experimentally, the model is implemented using a degenerate fermionic ${ }^{40} \mathrm{~K}$ cloud with $N=35(3) \times 10^{3}$ atoms [39] loaded into a three-dimensional optical lattice with a brick wall geometry [43]. Two equally populated magnetic sublevels of the $F=9 / 2$ hyperfine manifold 


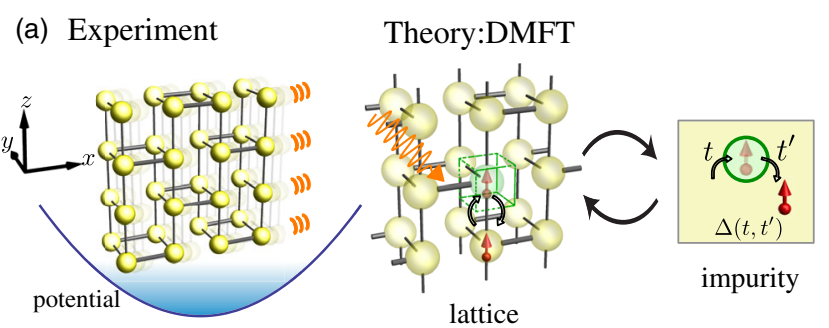

(b) $\hbar \Omega \gg U, J$

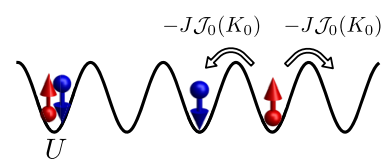

$\hbar \Omega \simeq U \gg J$

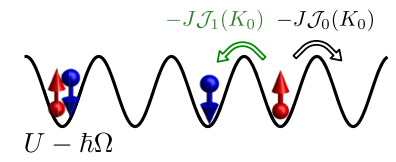

(c) $\Omega / 2 \pi(=3 \mathrm{kHz}) \gg U / h, J / h$

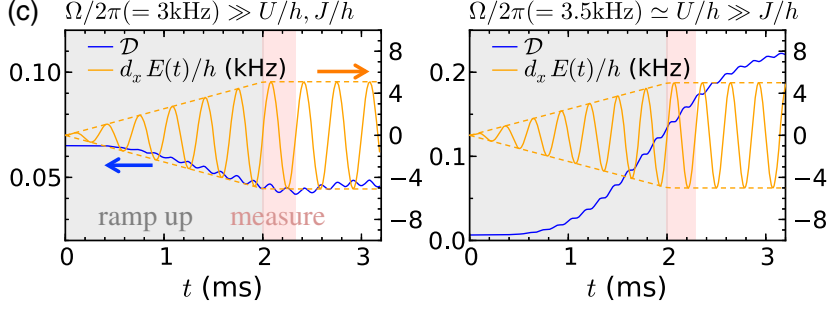

FIG. 1. (a) Experiment: Three-dimensional brick wall structure in a trapping potential. The driving is applied in the $x$ direction. Theory: DMFT mapping of the lattice system to an effective impurity problem. It is characterized by the hybridization function $\Delta\left(t, t^{\prime}\right)$, which mimics the hopping of particles to neighboring sites in the lattice system. (b) Schematic illustration of the different effective Hamiltonians. In the off-resonant regime $(\hbar \Omega \gg U, J)$, the interaction $U$ is unaffected while the hopping parameter $J$ is renormalized. In the near-resonant regime $(\hbar \Omega \approx U \gg J)$, the interactions are reduced to $U-\hbar \Omega$ and the hopping parameter depends on whether or not the tunneling process changes the number of doubly occupied sites. (c) DMFT simulations of the double occupation $\mathcal{D}$ in the off-resonant $\left(\Omega / 2 \pi=3 \mathrm{kHz}, U / h=750 \mathrm{~Hz}, J_{x}=200 \mathrm{~Hz}, J_{y}=J_{z}=40 \mathrm{~Hz}\right)$ and near-resonant $\left(\Omega / 2 \pi=U / h=3.5 \mathrm{kHz}, \quad J_{x}=200 \mathrm{~Hz}\right.$, $J_{y}=J_{z}=100 \mathrm{~Hz}$ ) regimes. As in the experiment, the driving field $E(t)$ is ramped up linearly during a period $t_{\text {ramp }}$ and $\mathcal{D}$ is measured just after the ramp and averaged over one period of the excitation $(T=(2 \pi / \Omega))$.

mimic the interacting spin-up and spin-down particles moving in the lowest band of the lattice. The time-periodic field in Eq. (1) is realized with a piezoelectric actuator moving the retroreflecting mirror of the lattice such that the $x$ position of the lattice is sinusoidally modulated [39]. It can be written as $E(t)=m A \Omega^{2} \sin (\Omega t)$, where $A$ is the displacement amplitude, $m$ the mass of the atoms, and $\Omega$ the angular driving frequency. A crossed dipole trap forms an overall harmonic confinement on top of the periodic potential generated by the lattice beams. The resulting atomic density distribution can be estimated in the loading lattice for an independently determined entropy [39].

On the theory side the same model is studied using nonequilibrium DMFT [33-35] (for details of the implementation, see Ref. [39]). DMFT is based on a self-consistent mapping to a quantum impurity model [Fig. 1(a)] and a local self-energy approximation, which becomes exact in the limit of infinite dimensions [44,45]. The periodic driving is incorporated by a Peierls factor in the hopping terms [35]. To solve the impurity problem, we use the noncrossing (NCA) and one-crossing (OCA) approximations [46-48]. It turns out that NCA is sufficient to describe the system in the present study [39]. The local density approximation (LDA) is employed to take into account the inhomogeneity of the cold atom system; i.e., we simulate the dynamics of homogeneous systems with different fillings and compute the average over the experimentally determined density distribution [39]. The comparison between theory and experiment is thus limited to timescales which are short enough that there is no significant redistribution of atoms within the trap [26].

The many-body dynamics can be captured by measuring the fraction of atoms on doubly occupied sites $\mathcal{D}=$ $2 / N \sum_{\mathbf{i}}\left\langle\hat{n}_{\mathbf{i} \uparrow} \hat{n}_{\mathbf{i} \downarrow}\right\rangle$ [39]. This observable indicates how the nature of the state changes when the effective on-site interaction changes or the system is driven in or out of strongly correlated regions. The value of $\mathcal{D}$ is averaged over the spatially inhomogeneous system and one driving cycle to distinguish the effective dynamics from micromotion $[49,50]$. Theoretical plots illustrating the full time evolution of $\mathcal{D}$ and the measurement protocol are shown in Fig. 1(c). In addition, DMFT calculations allow us to extract the local single-particle spectral function $A(\omega)$ and its particle (hole) occupation $N(\omega)[\bar{N}(\omega)]$ to investigate the driving induced couplings between many-body states [39].

In the off-resonant case $\hbar \Omega \gg U, W$, with $W=2 J_{x}+$ $4\left(J_{y}+J_{z}\right)$ denoting the free-fermion bandwidth, a highfrequency expansion to lowest order yields the effective Hamiltonian [51,52]

$$
\begin{aligned}
\hat{H}_{\text {off-res }}^{\text {eff }}= & -J_{x} \mathcal{J}_{0}\left(K_{0}\right) \sum_{\langle\mathbf{i}, \mathbf{j}\rangle_{x}, \sigma} \hat{c}_{\mathbf{i} \sigma}^{\dagger} \hat{c}_{\mathbf{j} \sigma} \\
& -J_{y, z} \sum_{\langle\mathbf{i}, \mathbf{j}\rangle_{y, z}, \sigma} \hat{c}_{\mathbf{i} \sigma}^{\dagger} \hat{c}_{\mathbf{j} \sigma}+U \sum_{\mathbf{i}} \hat{n}_{\mathbf{i} \downarrow} \hat{n}_{\mathbf{i} \uparrow} .
\end{aligned}
$$

This corresponds to a static Hubbard model with hopping in the $x$ direction renormalized by the zeroth-order Bessel function $\mathcal{J}_{0}\left(K_{0}\right)[53,54]$ which depends on the dimensionless driving amplitude $K_{0}=m A \Omega d_{x} / \hbar ; d_{x}$ denotes the distance of two neighboring sites in the $x$ direction. If we lower the driving frequency, higher order corrections to Eq. (2) have to be taken into account and reliable information on the evolution of the state can only be obtained by the combination of quantum simulations and time-dependent DMFT calculations.

For $U / W=1.1(1)$ we compare experimental [Fig. 2(a)] and theoretical [Fig. 2(b)] data for different drive frequencies in the off-resonant regime to first validate the effective Hamiltonian description according to Eq. (2). 

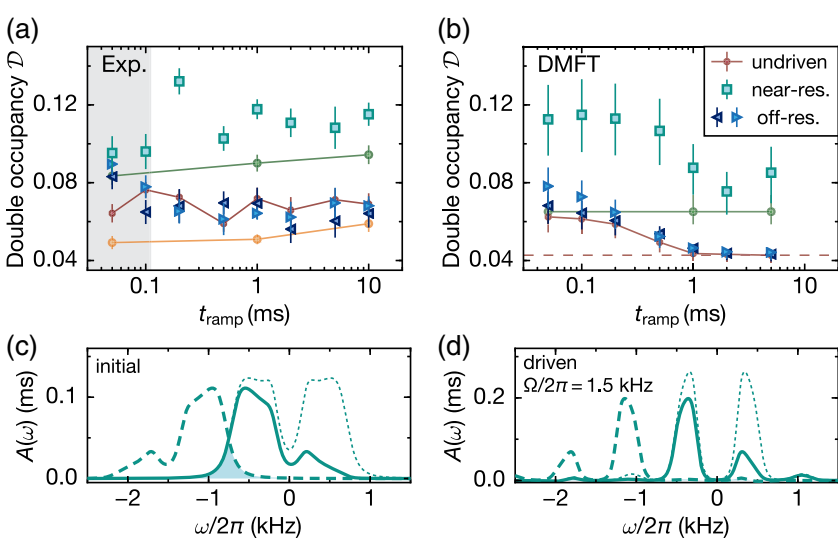

FIG. 2. Off-resonant driving in the Fermi-Hubbard model at $U / h=0.75(3)$ and $W / h=0.71(7) \mathrm{kHz}$. Experimental (a) and DMFT (b) data display $\mathcal{D}$ measured after ramping up the drive on different timescales. The legend applies to both plots. In the off-resonant case (triangle left: $\Omega / 2 \pi=5$, triangle right: $\Omega / 2 \pi=$ $3 \mathrm{kHz}) \mathcal{D}$ is suppressed, whereas an increase is observed when $\Omega / 2 \pi=1.5 \mathrm{kHz} \simeq(U+W) / h$ (squares). Solid lines show data taken in an undriven system. The upper and lower lines are reference values for holding in the initial $\left[J_{x} / h=193(34) \mathrm{Hz}\right]$ and final lattice $\left[J_{x}^{\text {eff }} / h=81(13) \mathrm{Hz}\right]$. The red line displays data taken after ramping the lattice depth from $J_{x}$ to $J_{x}^{\text {eff }}$ to mimic the driven data. The dashed red line indicates the saturation value reached at $t_{\text {ramp }}=5 \mathrm{~ms}$. Data in the shaded area of (a) are influenced by residual dynamics during detection and the finite bandwidth of the piezoelectric actuator. Error bars in (a) denote the standard error for 5 measurements and in (b) reflect the uncertainty of the entropy estimation in the experiment [39]. Panel (c) shows the local single-particle spectral function $A(\omega)$ (thin dashed) and its occupation $N(\omega)$ (solid) at $T / J_{x}=1.21$ in equilibrium at half-filling. The shaded area indicates the overlap between $N(\omega)$ and the hole occupation $\bar{N}(\omega-\Omega)$ shifted by the driving frequency $\Omega / 2 \pi=1.5 \mathrm{kHz}$ (dashed), corresponding to possible direct excitations. In (d) we plot the nonequilibrium spectra after ramping up the drive in $5 \mathrm{~ms}$.

We prepare an initial state with $\mathcal{D}=0.083(5)$ [39] and ramp up the modulation in different times $t_{\text {ramp }}$ to the final strength $\left[K_{0}=1.68(2)\right]$ before $\mathcal{D}$ is measured. If the renormalization to $J_{\text {eff }}$ is dominant, $\mathcal{D}$ is expected to be suppressed because $U / J_{\text {eff }}$ increases. This is verified by data taken in static lattices at the same entropy, with the hopping set once to the preparation parameter $\left(J_{x}\right.$, green $)$ and once to the effective value $\left[J_{x}^{\text {eff }}=J_{x} \mathcal{J}_{0}\left(K_{0}\right)\right.$, orange $]$. Theoretically, the same reference line for the preparation lattice is calculated (green) and for $J_{x} / h=81(12) \mathrm{Hz}$ the equilibration value of $\mathcal{D}$ is estimated by the adiabatic ramp of the hopping from $J_{x} / h=193(32) \mathrm{Hz}$ [39]. The cloud is loaded into a shallow lattice to achieve adiabatic preparation. However, in order to resolve the dynamics in the experiment, the tunneling energies in the starting lattice of the experiment are reduced. This leads to a gradual increase in $\mathcal{D}$ associated with the induced global density redistribution [39].
To test the validity of the effective Hamiltonian (2) we also simulate undriven systems in which the hopping amplitude is changed in time by a protocol which mimics the ramp-up of the driving amplitude $K_{0}$ in the effective Hamiltonian (red lines) [26,50]. For the large (off-resonant) driving frequencies, both theoretical and experimental results follow the trend of the effective Hamiltonian dynamics. The theoretical data clearly identify adiabatic timescales above $1 \mathrm{~ms}$ for reaching the equilibrium reference value which is consistent with the experimental data, although the latter are not fully conclusive due to the large scatter. In addition, the theoretical results show that the effective description is valid even when $t_{\text {ramp }}$ is comparable to a single cycle $[(2 \pi) / \Omega=0.2$ to $0.33 \mathrm{~ms}]$ of the modulation $[21,39]$.

By moving the drive frequency closer to resonances with the on-site interaction $U$ (see also Ref. [39]), we explore for which frequencies Eq. (2) still provides a good description of the system [39]. At $\Omega / 2 \pi=$ $1.5 \mathrm{kHz}$ the frequency is larger than $U$ and $W$ but comparable to $U+W$, which is the naively expected maximum energy of a double occupation excitation in the system. In this nontrivial regime both theory and experiment consistently predict a breakdown of the effective description.

Here $\mathcal{D}$ are created at short ramp times before decreasing again for longer times. Experimentally, times below $0.1 \mathrm{~ms}$ (shaded area) are difficult to access because of the finite bandwidth of the piezoelectric actuator and the detection time. From the theoretically obtained local single-particle spectral function one can see that direct excitations across the gap are possible because the bandwidth is broadened by the interaction [Fig. 2(c)]. This is most pronounced at short times as the effective bandwidth decreases due to the driving at longer times [Fig. 2(d)]. Interestingly, $\mathcal{D}$ does not decrease to the same values as for higher driving frequencies beyond $1 \mathrm{~ms}$. Despite a very similar final effective Hamiltonian, the final state is very different depending on the energy absorbed. This can be seen as nonadiabatic behavior that was confirmed by further studies in the off- and nearresonant regime [39]. Since the number of coupled states changes rapidly with driving frequency, $\mathcal{D}$ is very sensitive to $\Omega$ in this regime. We attribute the remaining deviations in the values of $\mathcal{D}$ between the $a b$ initio calculations and the experimental values to the systematic uncertainties on the input temperature and density profiles provided by the experiment [39].

A particularly appealing feature of Floquet engineering is the possible creation of effective Hamiltonians with terms that are difficult to realize in static systems. An example in the strong coupling regime is the near-resonantly driven system $(J \ll U \simeq \hbar \Omega)$, for which the effective Hamiltonian becomes $[20,50,55,56]$ 

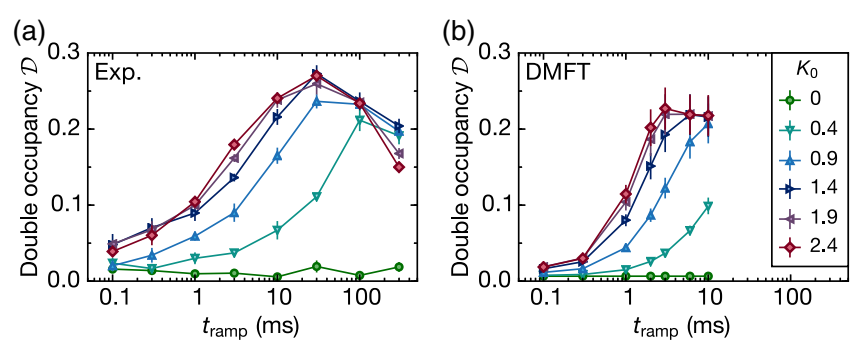

FIG. 3. Resonant driving in the Fermi-Hubbard model for $J_{x, y, z} / h=[200(50), 100(10), 100(10)]$ Hz. Experimentally measured (a) and theoretically simulated (b) $\mathcal{D}$ for different ramp times and driving strengths at resonance $[\Omega /(2 \pi)=3.5 \mathrm{kHz}$, $U / h=3.5(1) \mathrm{kHz}]$. Dynamics beyond $t_{\text {ramp }}=10 \mathrm{~ms}$ are influenced by trap effects and for $t_{\text {ramp }}=300 \mathrm{~ms}$ by heating [39] and is not considered in DMFT. Error bars are the same as in Fig. 2.

$$
\begin{aligned}
\hat{H}_{\text {res }}^{\text {eff }}= & -J_{x} \sum_{\substack{\mathbf{i} \in A, \cdot \\
\mathbf{j}=\mathbf{i}+e_{x}}}\left\{\left[\mathcal{J}_{0}\left(K_{0}\right) \hat{a}_{\mathbf{i j} \bar{\sigma}}+\mathcal{J}_{1}\left(K_{0}\right) \hat{b}_{\mathbf{i j} \bar{\sigma}}\right] \hat{c}_{\mathbf{i} \sigma}^{\dagger} \hat{c}_{\mathbf{j} \sigma}\right. \\
& + \text { H.c. }\}-J_{y, z} \sum_{\langle\mathbf{i}, \mathbf{j}\rangle_{y, z}, \sigma} \hat{a}_{\mathbf{i j}, \bar{\sigma}} \hat{c}_{\mathbf{i} \sigma}^{\dagger} \hat{c}_{\mathbf{j} \sigma} \\
& +(U-\hbar \Omega) \sum_{\mathbf{i}} \hat{n}_{\mathbf{i} \downarrow} \hat{n}_{\mathbf{i} \uparrow},
\end{aligned}
$$

as illustrated in the right panel of Fig. 1(b). In comparison to the static Hubbard model, the interaction is modified to the detuning from the drive $U^{\text {eff }}=U-\hbar \Omega$. The tunneling processes can be separated into two classes: (i) single particle tunneling processes that keep the number of double occupancies constant $\left[\hat{a}_{\mathbf{i} \mathbf{j} \bar{\sigma}}=\left(1-\hat{n}_{\mathbf{i} \bar{\sigma}}\right)\left(1-\hat{n}_{\mathbf{j} \bar{\sigma}}\right)+\hat{n}_{\mathbf{i} \overline{\overline{ }}} \hat{n}_{\mathbf{j} \bar{\sigma}}\right.$ and $\bar{\uparrow}=\downarrow]$, such that the interaction energy is irrelevant, and (ii) tunneling processes that increase or decrease the double occupancy by one unit $\left[\hat{b}_{\mathbf{i} \mathbf{j} \bar{\sigma}}=-\left(1-\hat{n}_{\mathbf{i} \bar{\sigma}}\right) \hat{n}_{\mathbf{j} \bar{\sigma}}+\hat{n}_{\mathbf{i} \bar{\sigma}}\left(1-\hat{n}_{\mathbf{j} \bar{\sigma}}\right)\right]$. Since one opposite spin particle is involved in the latter processes, these are density-dependent hoppings [49,50, 57-59] which make $\hat{H}_{\text {res }}^{\text {eff }}$ fundamentally different from a static Hubbard model.

In one set of measurements [Fig. 3(a) (experiment) and 3(b) (theory)] we initialize the cloud in a strongly interacting state $[U / W=2.9(3)]$ and ramp up the modulation while setting the frequency equal to the interaction $U$. For different driving strengths $K_{0}$ we measure the change of $\mathcal{D}$ for increasing ramp times [39]. From Eq. (3), it is expected that the $\mathcal{D}$ creation rate scales with $J_{x} \mathcal{J}_{1}\left(K_{0}\right)$ [39]. In the static case (green) the suppressed $\mathcal{D}$ reflects the strongly correlated regime. In the driven system a finite density-dependent term and reduced effective interactions result in an increase of $\mathcal{D}$. We find good agreement between theory and experiment. Both show the theoretically predicted creation of $\mathcal{D}$ scaling as $\mathcal{J}_{1}\left(K_{0}\right)$ averaged over the ramp-up in $K_{0}$ (see theoretical analysis in Ref. [39]). At longer times ( $t_{\text {ramp }}>10 \mathrm{~ms}$ ), the renormalized tunneling and interaction energies lead to a global redistribution of density, which manifests itself in an
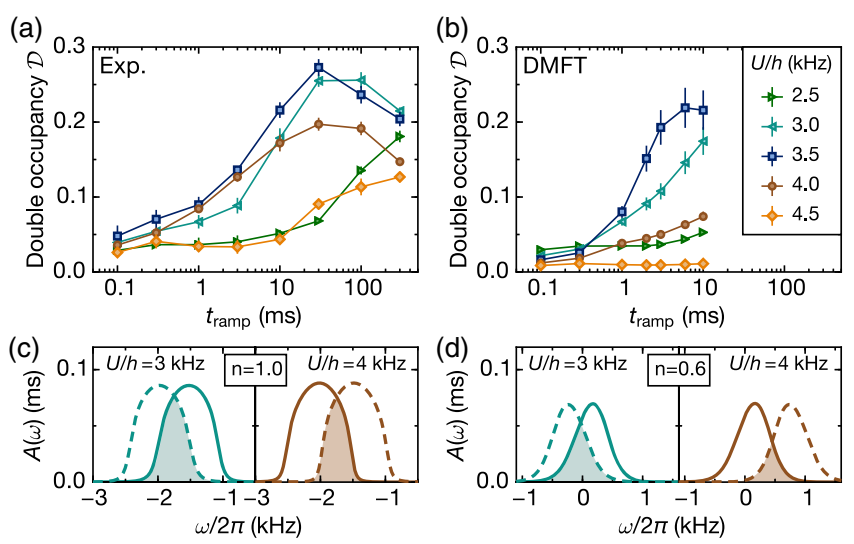

FIG. 4. Near-resonant driving in the Fermi-Hubbard model for $J_{x, y, z} / h=[200(50), 100(10), 100(10)] \mathrm{Hz}$ at fixed strength $K_{0}=1.44(2)$ and frequency $\Omega /(2 \pi)=3.5 \mathrm{kHz}$. Experimental (a) and numerical results (b) for $\mathcal{D}$ after different ramp times at interactions chosen symmetrically around the resonance. Dynamics beyond $t_{\text {ramp }}=10 \mathrm{~ms}$ are influenced by trap effects and for $t_{\mathrm{ramp}}=300 \mathrm{~ms}$ by heating [39] and is not considered in DMFT. Error bars are the same as in Fig. 2. In (c) and (d), the occupations of the lower Hubbard band (solid lines) at $T / J_{x}=$ 3.3 are shown for symmetric detunings. The shaded area indicates the overlap with the hole occupation (dashed) shifted by the driving frequency. The data in (c) are for half filling and in (d) for lower filling.

increase of $\mathcal{D}$. This trap induced effect cannot be captured by nonequilibrium DMFT. The following decrease at $300 \mathrm{~ms}$ is influenced by atom loss indicating the excitation of atoms to higher bands caused by absorption of energy from the drive [39].

In another set of measurements shown in Figs. 4(a) (experiment) and 4(b) (theory), we fix the strength $\left[K_{0}=\right.$ $1.44(2)]$ and drive frequency $(\Omega / 2 \pi=3.5 \mathrm{kHz})$ but change the interaction $U$ symmetrically around the resonance $(U / h=3.5 \mathrm{kHz})$ [39]. The far detuned data $(U / h=2.5$ and $4.5 \mathrm{kHz})$ show very low excitations of $\mathcal{D}$ for shorter ramp times, whereas in the near-resonant cases finite excitation rates appear. Experimentally, the curves at $U / h=3$ and $U / h=4 \mathrm{kHz}$ have a comparable excitation rate to the resonant case, but a lower saturation value for $U / h=4 \mathrm{kHz}$ indicates an asymmetry of the absorption with respect to the resonance frequency. In the DMFT data this asymmetry is already reflected in the creation rates. At half-filling, the rate is almost symmetric, consistent with the similar size of the overlap between the occupied states and the empty states shifted by the driving frequencies $U / h=3$ and $U / h=4 \mathrm{kHz}$ [Fig. 4(c)]. At lower fillings, since the bottom of the lower Hubbard band is more occupied, the overlap is reduced for $U / h=$ $4 \mathrm{kHz}$ [Fig. 4(d)], which results in the asymmetry. Overall, we find almost quantitative agreement between theory and experiment apart from the $U / h=4 \mathrm{kHz}$ case where the results are very sensitive to the exact Hubbard parameters, which is not represented in the error bar of the theoretical 
calculation. The longer ramp times $\left(t_{\text {ramp }}>10 \mathrm{~ms}\right)$, only measured in the experiment, reveal an initial increase in $\mathcal{D}$ for all detunings followed by a decrease for small detunings. This dynamics is again resulting from trap induced effects, technical heating and coupling to higher bands [26,39].

In this work we demonstrated how basic models of nonequilibrium, strongly correlated systems can be explored experimentally and numerically to reveal their fundamental dynamics. New insights into pump-probe experiments in solid state physics can be gained by looking at the many-body dynamics of these strongly driven models $[35,50]$. Furthermore, the cross validation of the presented methods reveals the driving regimes where the physics is described by a desired effective Hamiltonian. In both experiment and theory, different model Hamiltonians can be realized including a fully tunable Heisenberg and $t-J$ model $[50,60,61]$ or anyonic Hubbard models and dynamical gauge fields resulting from occupation dependent Peierls phases [55,62-67].

We thank W. Zwerger for encouraging this collaboration, and J. Coulthard, H. Gao, D. Golež, D. Jaksch, M. Schüler, and K. Viebahn for helpful discussions. We acknowledge the Swiss National Science Foundation (Projects No. 169320 and No. 182650, NCCR-QSIT and NCCRMARVEL), the Swiss State Secretary for Education, Research and Innovation Contract No. 15.0019 (QUIC), ERC advanced grant TransQ (Project No. 742579) and ERC consolidator grant Modmat (Project No. 724103) for funding. The DMFT calculations have been performed on the Beo04 and Beo05 clusters at the University of Fribourg.

[1] R. P. Feynman, Int. J. Theor. Phys. 21, 467 (1982).

[2] I. M. Georgescu, S. Ashhab, and F. Nori, Rev. Mod. Phys. 86, 153 (2014).

[3] I. Bloch, J. Dalibard, and S. Nascimbène, Nat. Phys. 8, 267 (2012).

[4] L. Tarruell and L. Sanchez-Palencia, C.R. Phys. 19, 365 (2018).

[5] R. Jördens, L. Tarruell, D. Greif, T. Uehlinger, N. Strohmaier, H. Moritz, T. Esslinger, L. De Leo, C. Kollath, A. Georges, V. Scarola, L. Pollet, E. Burovski, E. Kozik, and M. Troyer, Phys. Rev. Lett. 104, 180401 (2010).

[6] B. Sciolla, A. Tokuno, S. Uchino, P. Barmettler, T. Giamarchi, and C. Kollath, Phys. Rev. A 88, 063629 (2013).

[7] J. Imriška, M. Iazzi, L. Wang, E. Gull, D. Greif, T. Uehlinger, G. Jotzu, L. Tarruell, T. Esslinger, and M. Troyer, Phys. Rev. Lett. 112, 115301 (2014).

[8] A. Golubeva, A. Sotnikov, and W. Hofstetter, Phys. Rev. A 92, 043623 (2015).

[9] R. A. Hart, P. M. Duarte, T.-L. Yang, X. Liu, T. Paiva, E. Khatami, R. T. Scalettar, N. Trivedi, D. A. Huse, and R. G. Hulet, Nature (London) 519, 211 (2015).

[10] E. Cocchi, L. A. Miller, J. H. Drewes, M. Koschorreck, D. Pertot, F. Brennecke, and M. Köhl, Phys. Rev. Lett. 116, 175301 (2016).
[11] A. Mazurenko, C. S. Chiu, G. Ji, M. F. Parsons, M. KanászNagy, R. Schmidt, F. Grusdt, E. Demler, D. Greif, and M. Greiner, Nature (London) 545, 462 (2017).

[12] D. Nicoletti and A. Cavalleri, Adv. Opt. Photonics 8, 401 (2016).

[13] M. Mitrano, A. Cantaluppi, D. Nicoletti, S. Kaiser, A. Perucchi, S. Lupi, P. Di Pietro, D. Pontiroli, M. Riccò, S. R. Clark, D. Jaksch, and A. Cavalleri, Nature (London) 530, 461 (2016).

[14] N. Goldman, J. C. Budich, and P. Zoller, Nat. Phys. 12, 639 (2016).

[15] A. Eckardt, Rev. Mod. Phys. 89, 011004 (2017).

[16] T. Oka and S. Kitamura, Annu. Rev. Condens. Matter Phys. 10, 387 (2019).

[17] N. Goldman and J. Dalibard, Phys. Rev. X 4, 031027 (2014).

[18] M. Bukov, L. D’Alessio, and A. Polkovnikov, Adv. Phys. 64, 139 (2015).

[19] T. Mikami, S. Kitamura, K. Yasuda, N. Tsuji, T. Oka, and H. Aoki, Phys. Rev. B 93, 144307 (2016).

[20] M. Bukov, M. Kolodrubetz, and A. Polkovnikov, Phys. Rev. Lett. 116, 125301 (2016).

[21] M. Eckstein, J. H. Mentink, and P. Werner, arXiv:1703 .03269 .

[22] K. Singh, K. M. Fujiwara, Z. A. Geiger, E. Q. Simmons, M. Lipatov, A. Cao, P. Dotti, S. V. Rajagopal, R. Senaratne, T. Shimasaki, M. Heyl, A. Eckardt, and D. M. Weld, arXiv: 1809.05554.

[23] T. Mori, T. N. Ikeda, E. Kaminishi, and M. Ueda, J. Phys. B 51, 112001 (2018).

[24] R. Moessner and S. L. Sondhi, Nat. Phys. 13, 424 (2017).

[25] A. Herrmann, Y. Murakami, M. Eckstein, and P. Werner, Europhys. Lett. 120, 57001 (2017).

[26] M. Messer, K. Sandholzer, F. Görg, J. Minguzzi, R. Desbuquois, and T. Esslinger, Phys. Rev. Lett. 121, 233603 (2018).

[27] M. Weinberg, C. Ölschläger, C. Sträter, S. Prelle, A. Eckardt, K. Sengstock, and J. Simonet, Phys. Rev. A 92, 043621 (2015).

[28] C. Sträter and A. Eckardt, Z. Naturforsch. A 71, 909 (2016).

[29] M. Reitter, J. Näger, K. Wintersperger, C. Sträter, I. Bloch, A. Eckardt, and U. Schneider, Phys. Rev. Lett. 119, 200402 (2017).

[30] S. Lellouch, M. Bukov, E. Demler, and N. Goldman, Phys. Rev. X 7, 021015 (2017).

[31] J. Näger, K. Wintersperger, M. Bukov, S. Lellouch, E. Demler, U. Schneider, I. Bloch, N. Goldman, and M. Aidelsburger, arXiv:1808.07462.

[32] T. Boulier, J. Maslek, M. Bukov, C. Bracamontes, E. Magnan, S. Lellouch, E. Demler, N. Goldman, and J. V. Porto, Phys. Rev. X 9, 011047 (2019).

[33] A. Georges, G. Kotliar, W. Krauth, and M. J. Rozenberg, Rev. Mod. Phys. 68, 13 (1996).

[34] J. K. Freericks, V. M. Turkowski, and V. Zlatić, Phys. Rev. Lett. 97, 266408 (2006).

[35] H. Aoki, N. Tsuji, M. Eckstein, M. Kollar, T. Oka, and P. Werner, Rev. Mod. Phys. 86, 779 (2014).

[36] H. U. R. Strand, D. Golež, M. Eckstein, and P. Werner, Phys. Rev. B 96, 165104 (2017). 
[37] M. Ligges, I. Avigo, D. Golež, H. U. R. Strand, Y. Beyazit, K. Hanff, F. Diekmann, L. Stojchevska, M. Kalläne, P. Zhou, K. Rossnagel, M. Eckstein, P. Werner, and U. Bovensiepen, Phys. Rev. Lett. 120, 166401 (2018).

[38] N. Tsuji, P. Barmettler, H. Aoki, and P. Werner, Phys. Rev. B 90, 075117 (2014).

[39] See Supplemental Material Secs. I-IV at http://link.aps.org/ supplemental/10.1103/PhysRevLett.123.193602, for more details, which includes Refs. [40-42].

[40] F. Peronaci, M. Schiró, and O. Parcollet, Phys. Rev. Lett. 120, 197601 (2018).

[41] T. Uehlinger, G. Jotzu, M. Messer, D. Greif, W. Hofstetter, U. Bissbort, and T. Esslinger, Phys. Rev. Lett. 111, 185307 (2013).

[42] J. Oitmaa, C. Hamer, and W. Zheng, Series Expansion Methods for Strongly Interacting Lattice Models (Cambridge University Press, Cambridge, England, 2006).

[43] L. Tarruell, D. Greif, T. Uehlinger, G. Jotzu, and T. Esslinger, Nature (London) 483, 302 (2012).

[44] W. Metzner and D. Vollhardt, Phys. Rev. Lett. 62, 324 (1989).

[45] E. Mueller-Hartmann, Z. Phys. B 74, 507 (1989).

[46] H. Keiter and J. Kimball, J. Appl. Phys. 42, 1460 (1971).

[47] T. Pruschke and N. Grewe, Z. Phys. B 74, 439 (1989).

[48] M. Eckstein and P. Werner, Phys. Rev. B 82, 115115 (2010).

[49] R. Desbuquois, M. Messer, F. Görg, K. Sandholzer, G. Jotzu, and T. Esslinger, Phys. Rev. A 96, 053602 (2017).

[50] F. Görg, M. Messer, K. Sandholzer, G. Jotzu, R. Desbuquois, and T. Esslinger, Nature (London) 553, 481 (2018).

[51] D. H. Dunlap and V. M. Kenkre, Phys. Rev. B 34, 3625 (1986).

[52] A. Eckardt, C. Weiss, and M. Holthaus, Phys. Rev. Lett. 95, 260404 (2005).
[53] H. Lignier, C. Sias, D. Ciampini, Y. Singh, A. Zenesini, O. Morsch, and E. Arimondo, Phys. Rev. Lett. 99, 220403 (2007).

[54] A. Zenesini, H. Lignier, D. Ciampini, O. Morsch, and E. Arimondo, Phys. Rev. Lett. 102, 100403 (2009).

[55] A. Bermudez and D. Porras, New J. Phys. 17, 103021 (2015).

[56] A. P. Itin and M. I. Katsnelson, Phys. Rev. Lett. 115, 075301 (2015).

[57] R. Ma, M. E. Tai, P. M. Preiss, W. S. Bakr, J. Simon, and M. Greiner, Phys. Rev. Lett. 107, 095301 (2011).

[58] F. Meinert, M. J. Mark, K. Lauber, A. J. Daley, and H.-C. Nägerl, Phys. Rev. Lett. 116, 205301 (2016).

[59] W. Xu, W. Morong, H. Y. Hui, V. W. Scarola, and B. DeMarco, Phys. Rev. A 98, 023623 (2018).

[60] J. R. Coulthard, S. R. Clark, and D. Jaksch, Phys. Rev. B 98, 035116 (2018).

[61] J. H. Mentink, K. Balzer, and M. Eckstein, Nat. Commun. 6, 6708 (2015).

[62] T. Keilmann, S. Lanzmich, I. McCulloch, and M. Roncaglia, Nat. Commun. 2, 361 (2011).

[63] S. Greschner and L. Santos, Phys. Rev. Lett. 115, 053002 (2015).

[64] L. Cardarelli, S. Greschner, and L. Santos, Phys. Rev. A 94, 023615 (2016).

[65] C. Sträter, S. C. L. Srivastava, and A. Eckardt, Phys. Rev. Lett. 117, 205303 (2016).

[66] S. Greschner, G. Sun, D. Poletti, and L. Santos, Phys. Rev. Lett. 113, 215303 (2014).

[67] L. Barbiero, C. Schweizer, M. Aidelsburger, E. Demler, N. Goldman, and F. Grusdt, Sci. Adv..5, eaav7444 (2019). 